\title{
The Persuasiveness of Web-Based Alcohol Interventions
}

\author{
Tuomas Lehto and Harri Oinas-Kukkonen \\ University of Oulu, Department of Information Processing Science \\ Rakentajantie 3, FIN-90570 Oulu, Finland \\ \{Tuomas. Lehto, Harri. Oinas-Kukkonen\}@oulu.fi
}

\begin{abstract}
There are a variety of Web-based alcohol interventions that may reach problem drinkers, who would not otherwise participate in conventional treatment. Web-based alcohol interventions vary greatly in level of finesse: some offer static self-help materials, whereas some sites have highly interactive content and persuasive features embedded. In this study, six Web-based alcohol interventions were evaluated based on a framework for evaluating and designing persuasive systems. This study demonstrates the potential lack of persuasive features on Web-based alcohol interventions sites. Important primary task support elements, such as tailoring and personalization, were used tenuously throughout the sites. The dialogue support demonstrated throughout the sites was average. All evaluated sites successfully demonstrated trustworthiness, expertise, and surface credibility. Many of the evaluated sites were lacking in the social support category. In general, the authors suggest that the persuasive system qualities should be considered concurrently with the feasibility and effectiveness for studying technology-based interventions.
\end{abstract}

Keywords: Web-based, alcohol, intervention, persuasive technology, PSD model.

\section{Introduction}

Harm caused by excess alcohol consumption is a major public health concern throughout the developed world [15]. Alcohol is causally related to more than 60 different medical conditions, and $4 \%$ of the global burden of disease is estimated to be attributable to alcohol [26]. Heavy and binge drinking is associated with a variety of physical and mental health problems [15].

There are different types of behavioral and pharmacological interventions available for treating alcohol dependence [26]. The terms addiction and dependence are being used interchangeably, and it is unlikely that there is going to be a consensus on the definition of and distinction between these terms [30]. Despite the existence and availability of preventive measures both at the individual and population levels, alcohol problems present a major challenge to medicine and public health [26].

Technology-based interventions related to, for example, nutrition [19], physical exercise [10], [17], smoking [3], [27], alcohol consumption [2], [6], [14], stress management [31], and depression [4], [5] may delay or prevent the onset of a variety of medical problems, and improve the quality of life [11]. A primary goal of self-care interventions is the encouragement of an individual's behavior change, which requires knowledge sharing, education, and understanding of the condition [29]. 
Computer-tailored health interventions vary in terms of level of sophistication. At one end of the scale are computer-assisted risk or health assessments. These are usually brief interventions, providing personalized feedback to the user immediately online or emailed at a later time. At the other end of the scale are longer-term, more complex computer-tailored health programs, which have the potential for covering multiple or difficult-to-influence behaviors. [16]

Relatively few studies have discussed persuasive functionalities in practice. The goal of this study is to contribute to this discussion. Furthermore, the rationale for this study is our concern that the majority of digital interventions may have been developed without a proper insight into what constitutes a successful persuasive system.

The predominant challenge in designing and evaluating technology-enabled interventions, for a variety of health and behavior related issues, is how best to merge theories and approaches from psychology, healthcare, education, software design, and other relevant disciplines [8].

In this study, six Web-based alcohol interventions were evaluated and their persuasiveness was studied utilizing a framework for evaluating and designing persuasive systems. On a more detailed level, we explored how the selected sites used/adhered to the persuasive systems principles and techniques defined in the PSD model [22].

\section{Background}

There is a growing body of research on the use of the Internet, and the Web, as a means of delivering treatment. Internet interventions are typically focused on behavioral issues, with the goal of instituting behavior change and subsequent symptom improvement. They are usually self-paced, tailored to the user, interactive, and make use of the multimedia. Individuals who use this kind of intervention may overcome many of the obstacles to obtaining traditional care because they can seek such treatments at any time, any place, and often at significantly reduced cost. [25]

In their qualitative review, Copeland and Martin [6] concluded that brief interventions have been the most commonly reported, probably because there is little complexity involved in developing a minimal screener/feedback program in comparison to a fully automated therapy. They also pointed out that the complexity, quality and content of these interventions vary vastly.

There are several important decisions to be made when providing interventions for a variety of alcohol-related problems. For instance, the type, setting, and intensity of the intervention must be tailored to individuals' needs. For some heavy drinkers (without evidence of severe alcohol dependence), an intervention aimed at the reduction of drinking to moderate levels of consumption may suffice. By contrast, the goals of treatment for chronically alcohol-dependent people and high-level drinkers typically include complete abstinence from alcohol, and promotion of long-term recovery. [26]

According to Koski-Jännes et al. [13], there are three groups of problematic drinkers: 1) hazardous or risk drinkers who consume alcohol above recommended limits without any noticeable harm yet, 2) harmful drinkers who have experienced some physical, social or psychological harm without meeting the criteria of dependence, and 3) alcohol abusing or dependent drinkers who meet these criteria and sustain consuming alcohol regardless of substantial negative consequences. Internet-based 
self-assessments are meant to serve all of these groups, but they are effective mainly in reducing alcohol consumption among hazardous and harmful drinkers. Alcoholdependent drinkers require a different approach regarding support and treatment. Internet-based self-assessments appear to increase interest in various forms of selfhelp, especially in Internet-based interactive services for heavy drinkers [12]. There are many advantages in these services: high accessibility, affordability, and discretion. Discretion is a very important factor for individuals wanting to avoid being labeled as problem drinkers or alcoholics. [13]

In their recent systematic review, Bewick et al. [1] found inconsistent evidence on the effectiveness of electronic screening and brief intervention for alcohol use. However, their notion is that Web-based interventions are generally well received. Riper et al. [24] report that the few available evaluation studies of Web-based interventions for problem drinking, most of which involved student populations, have reported promising results.

Digital and fully automated health behavior interventions have the potential of high reach and low cost. A recent meta-analysis of 75 randomized controlled trials provided support for their effectiveness in changing knowledge, attitudes, and behavior in the health promotion area [23]. The benefits of persuasive interventions are crucially dependent on the quality and relevance of the technology and inference algorithms. However, user studies that look at the long-term effectiveness of persuasive technology hardly exist. [11]

\section{Evaluating Persuasive Systems}

Research on persuasive technology has been introduced relatively recently [9]. Persuasive systems may be defined as computerized software or information systems designed to reinforce, change or shape attitudes or behaviors or both without using coercion or deception [21]. Persuasive technologies can be used in different domains, for example in education, safety and healthcare [9]. Information technology always influences people's attitudes and behavior in one way or another thus all information technology is a persuasive system to some degree [22].

Oinas-Kukkonen and Harjumaa [20], [22] have conceptualized a framework for designing and evaluating persuasive systems, known as the Persuasive Systems Design (PSD) model. The PSD presents a way to analyze and evaluate the persuasion context and related techniques. Persuasion context analysis includes recognizing the intent (persuader, change type) and the event (use context, user context, and technology context) for persuasion, and recognizing the strategies (message, route) in use. In the PSD model, the categories for persuasive system principles are: primary task support (supporting the user's primary task), dialogue support (supporting the interaction between the user and the system), system credibility (the more credible the system is, the more persuasive it is), and social support (the system motivates users by leveraging social influence).

Primary task support. This category addresses the target behaviors. For this category, seven principles are employed: reduction, tunneling, tailoring, personalization, self-monitoring, simulation, and rehearsal. 
Dialogue support. This category deals with the feedback that the system offers in guiding the user to reach the intended behavior. Seven design principles are employed for providing dialogue support: praise, rewards, reminders, suggestion, similarity, liking, and social role.

System credibility support. Credibility (or ethos) is a persuasive element. The PSD model describes seven design principles for supporting system credibility: trustworthiness, expertise, surface credibility, real world feel, authority, third party endorsements, and verifiability.

Social support. This category describes how to design the system so that it motivates users by leveraging social influence. The model operates with seven design principles for providing social support: social learning, social comparison, normative influence, social facilitation, cooperation, compensation, and recognition.

In this study, the PSD framework will be applied for identifying the persuasive techniques that have been incorporated into the Web-based alcohol interventions.

\section{Research Setting}

Web-based alcohol intervention sites were extensively searched (during November 2008January 2009) from the Internet using public search engines (e.g., Google, Yahoo!), using the following search terms: alcohol(ism), help, stop, quit, recovery, drink(ing), consumption, abstinence, intervention, addiction, dependence, Web, internet, self-help. The search method is by no means exhaustive. For instance, the search phrase "alcoholism recovery" yields tens of thousands of hits on Google alone. In our view, going through all the possible combinations and variations of the search terms is nearly impossible and redundant. We did not limit ourselves to operate solely based on search engines, but also searched relevant literature to find the intervention sites that fell within the scope of this study. The literature was fetched from databases, such as EBSCOhost, Google Scholar, ISI Web of Knowledge, PubMed, and Scopus.

The inclusion criteria for a Web-based intervention portal were:

- it targets reduction of alcohol consumption or supports abstinence from alcohol,

- it is open-access, free of charge (may require registration), ready-to-use,

- it offers interactive functionality, and

- it is in English, Finnish, or Swedish.

An iterative search process (see Figure 1) yielded six Web-based alcohol intervention sites for further evaluation. Four of the intervention portals were in English, one was in Finnish, and one in Swedish. The three languages were chosen based on the authors' language skills.

Alcohol Help Center 2.0 (http://www.alcoholhelpcenter.net, later A1) is intended for individuals who are concerned about their problem drinking and who might want to cut down or quit drinking. The website is produced by Evolution Health (V-CC Systems), which is a privately held corporation. 


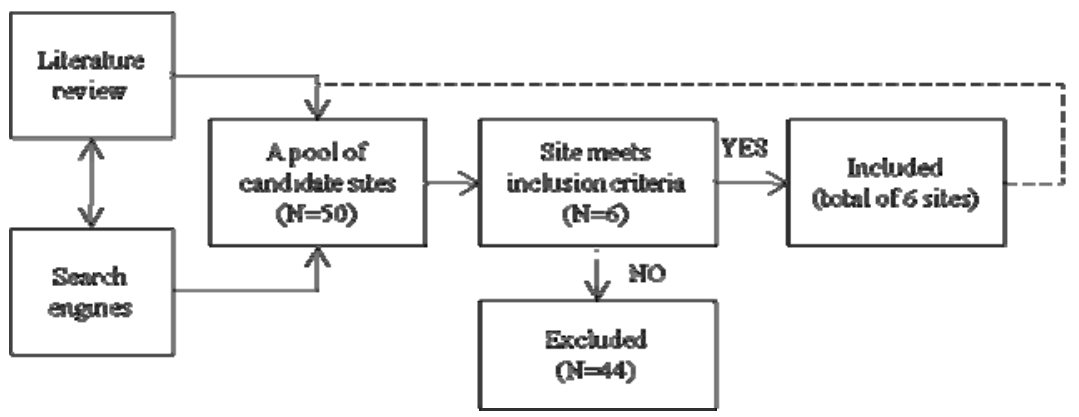

Fig. 1. The search process of the study

Control Your Drinking Online (http://www.acar.net.au/online.asp, A2) is a Webbased self-change program. The website is intended to assist and promote self-change amongst people who drink a lot and wish to reduce their alcohol intake. The Website is produced by the Australian Centre for Addiction Research (ACAR).

Down Your Drink (http://www.downyourdrink.org.uk, A3) has been designed to give people the information they need to make choices about the role alcohol plays in their life. The content of the website has been written by Stuart Linke and Jim McCambridge and produced by Net Impact. The charity Alcohol Concern manages the website.

Sober24 (http://www.sober24.com, A4) is a website for individuals who are recovering from alcohol or drug addiction. The site is also intended for an addicted person's loved ones.

Alkoholhjälpen (http://www.alkoholhjalpen.se, A5) is a website funded by the Swedish National Institute of Public Health. The self-help site divides into two parts; one part is intended for people who are worried about their drinking habits, the other part is for people who are worried about somebody else's drinking (e.g., a friend, a relative, a parent).

Jeppe Juomapäiväkirja (http://www.paihdelinkki.fi/jeppe/index.php, A6) is a Finnish website for people who are willing to reduce or quit drinking. The organization behind the service is the A-Clinic Foundation. The A-Clinic Foundation operates to reduce alcohol, drug and other addiction problems. A6 is a Finnish version of the Dutch MinderDrinken.

The portals were evaluated based on the principles for persuasive system content and functionality presented in the PSD model. The model does not suggest that all systems should always implement all of the features described in it. However, due to the nature of this study, and as the quality of the implemented features varied tremendously, we found it appropriate to use a scoring system. The goal was not to compare the sites against each other since they vary greatly in terms of complexity, content and quality. The aim is to demonstrate this diversity, and pinpoint the possible mutual and individual highlights/shortcomings in persuasive system features on the intervention sites under inspection. 


\section{Results}

\subsection{Persuasion Context}

\subsubsection{The Intent}

\section{Persuader}

The persuaders behind the interventions appear to be different kinds of organizations. Five of the six sites clearly state the background organization. The persuader behind one of the sites (A4) remains unclear.

\section{Change Type}

All of the evaluated sites ultimately aim at individuals' reduced alcohol consumption and/or quitting drinking. Self-help, personal goal-setting, and motivation play an important role in the change process in all of these sites.

The majority of the sites do not explicitly state whether they target change in individuals' behavior or attitude. However, it is relatively safe to assume that the sites favor behavior change over attitude change. In our view, the persuasiveness of the evaluated sites lacks in fostering individuals' long-term behavior change, let alone true attitudinal change, which may be the most difficult to achieve [22].

\subsubsection{The Event}

\section{Use Context}

The use context is described on a very general level on all of the evaluated sites. The intended user groups are hard to identify from such statements as: "Are you concerned about problem drinking? Do you think you might need to cut down or are you thinking about quitting?" (A1), or "If you are someone who wants to think seriously about your level of alcohol consumption - then this website may be for you" (A3). It would appear that the sites fail to recognize different user groups with varying needs, leading to a questionable one-fits-all approach. Tørning and Oinas-Kukkonen [28] argue that systems are often designed to persuade an individual at a system-to-user level to resolve a user-in-society issue.

It seems that Web-based alcohol interventions a) without predefined user groups and b) with minimal tailoring for the groups, cannot offer much more than educational content with minimal effect on users' behavior. For example, differences in gender and culture might also play a role in persuasion [28].

\section{User Context}

What is specific to the users of the evaluated Web-based alcohol intervention sites is that they are heavy drinkers or people who are concerned about their drinking. There are possible limitations, e.g. pregnant women and people under 18 should not participate in the interventions. In addition, the interventions are not meant to replace the advice of a physician or other health care provider.

\section{Technology Context}

All of the evaluated interventions are Web-based. Most of them work with a basic Web browser, with no additional plug-ins or installations required. Furthermore, the content presented is relatively small in size (except in A5), so that users with lower 
bandwidth Internet connections (and/or older computers) can use the sites without great difficulties. Seemingly, the technology context is similar in all of the interventions. Yet, a more detailed inspection reveals differences between the sites.

Alkoholhjälpen (A5) takes advantage of Adobe Flash software. In fact, the majority of the site content is delivered in Flash. This may increase the loading times substantially, thus making the site less usable for users with slower connections. However, some users may favor professionally designed Flash content over traditional HTML-based presentations. There is also some Flash content on Down Your Drink (A3) site. Control Your Drinking Online offers an interesting approach. The self-help material ("Treatment modules") is delivered to the user in printable PDF (Portable Document Format) files. This approach eliminates important persuasive features such as interactivity and tailoring, but still might suit some users. None of the sites seem to be designed for mobile use. Alcohol Help Center has an SMS (text messaging) service providing tips.

\subsubsection{The Strategy}

The strategy harnessed for persuading the users is very hard to determine from the studied web-based alcohol interventions. Some of the sites state clearly the underpinning methods or procedures, e.g. "The exercises and the information are taken from evidence-based methods such as cognitive behavioral therapy, motivational interviewing and relapse prevention" (A5, translation by the authors).

Message

Presumably, the wider the content the more embedded messages and arguments are likely to be presented to the user. Some of the sites offer a very broad content (A3, A5) whereas others rely on more compact presentation. It has to be noted that the total number of embedded arguments may not necessarily be relevant, compared to the manner in which they are presented.

\section{Route}

The embedded arguments seem to try to appeal to users' emotions and logic in all of the sites. All of the sites appear to target the individuals' behavior change through a set of arguments, instead of using one convincingly strong argument only (direct vs. indirect persuasion).

\subsection{Persuasion Techniques}

\subsubsection{Primary Task Support}

The sites varied greatly in persuasive system characteristics. Three of the sites (A2, A4 and A6) received a relatively low total score on the primary task support category. Reduction and tunneling were applied in five out of six sites. Surprisingly, tailoring (i.e. different content for different user groups) was applied only in one of the six sites. Furthermore, only low-level personalization was used in all of the sites. All of the sites received low scores on simulation and rehearsal, whereas they all showed self-monitoring (e.g. a drinking diary) functionality. Only one (A5) of the evaluated sites utilized all principles from the primary task support dimension. 
Table 1. Primary task support scores

$\begin{array}{lllllll}\text { Primary task } & \text { A1 } & \text { A2 } & \text { A3 } & \text { A4 } & \text { A5 } & \text { A6 } \\ \text { Reduction } & + & - & +++ & ++ & +++ & + \\ \text { Tunneling } & ++ & + & +++ & - & +++ & (+) \\ \text { Tailoring } & - & - & - & - & + & - \\ \text { Personalization } & (+) & + & (+) & + & ++ & (+) \\ \text { Self-monitoring } & +++ & (+) & ++ & ++ & +++ & ++ \\ \text { Simulation } & - & (+) & + & - & ++ & - \\ \text { Rehearsal } & + & (+) & ++ & - & + & (+)\end{array}$

Regardless of the different approaches in each site, it is alarming that many of the users' primary task support did not seem to be utilized fully. The lack of tailoring (and personalization) is a severe shortcoming on a health behavior change website. The interventions that direct participants to relevant, individually tailored materials appear to have longer Web site session times per visit and more visits per person [29].

\subsubsection{Dialogue Support}

Web-based systems can give immediate feedback, which makes it more likely that the information is matched to the respondent's level of awareness, beliefs and motivations at that particular time. Web-delivered tailored feedback resembles interpersonal counseling, which may enhance the effect of the intervention. [18]

There was variance on the feedback the sites offered in guiding the user to reach the intended behavior. The majority of the interventions demonstrated praise (e.g. user is presented with a positive message upon completing a specific task). However, the praise was usually presented very meagerly. None of the sites offered virtual rewards, such as pictures, sounds, or special content. Half of the intervention sites used reminders (e.g. daily or weekly e-mails). Every site presented suggestions to the user. Since the sites were not directed to a certain demographic, all of them used general language in presenting the information.

Table 2. Dialogue support scores

$\begin{array}{lllllll}\text { Dialogue } & \text { A1 } & \text { A2 } & \text { A3 } & \text { A4 } & \text { A5 } & \text { A6 } \\ \text { Praise } & (+) & (+) & - & + & ++ & - \\ \text { Rewards } & (+) & (+) & - & - & - & - \\ \text { Reminders } & +++ & - & ++ & ++ & - & (+) \\ \text { Suggestion } & + & (+) & + & + & ++ & + \\ \text { Similarity } & + & + & + & + & + & + \\ \text { Liking } & + & + & ++ & + & ++(+) & + \\ \text { Social role } & +++ & - & + & - & ++ & +\end{array}$

A system that is visually attractive is likely to be more persuasive. All of the sites scored on the liking principle. Most of the systems adopted a social role (e.g. to facilitate communication between users and health specialists). Overall, the dialogue support demonstrated throughout the sites was average. 


\subsubsection{Credibility Support}

All evaluated sites successfully demonstrated trustworthiness (e.g. the presented information is truthful, fair and unbiased) and expertise. In addition, they showed surface credibility (6/6 sites) and low-level real-world feel (5/6 sites).

Table 3. Credibility support scores

Credibility
Trustworthiness
Expertise
Surface credibility
Real-world feel
Authority
$3^{\text {rd }}$ party endorsements
Verifiability

$\begin{array}{llllll}\text { A1 } & \text { A2 } & \text { A3 } & \text { A4 } & \text { A5 } & \text { A6 } \\ ++ & ++ & ++ & + & ++ & ++ \\ ++ & ++ & ++ & + & ++ & + \\ ++ & + & ++ & + & ++ & ++ \\ + & + & + & - & + & + \\ - & + & - & - & +++ & + \\ - & - & + & + & - & - \\ ++ & - & ++ & + & + & +\end{array}$

Half of the sites referred to an authority, and two presented third party endorsements. Verifiability was quite low on the majority of the interventions. As a conclusion, none of the six sites had any remarkable problems with credibility issues.

\subsubsection{Social Support}

The greatest differences between the six Web-based alcohol intervention sites were observed in the social support category. Half of the sites (A1, A4, A5) offered a variety of social support functionality, whereas the other half lacked social support completely. The most versatile sites in terms of social support were A4 and A5, which took advantage of 6 out of 7 principles from the social support category.

Table 4. Social support scores

$\begin{array}{lllllll}\text { Social support } & \text { A1 } & \text { A2 } & \text { A3 } & \text { A4 } & \text { A5 } & \text { A6 } \\ \text { Social learning } & ++ & - & - & +++ & ++ & - \\ \text { Social comparison } & +++ & - & - & +++ & ++ & - \\ \text { Normative influence } & ++ & (+) & (+) & +++ & ++ & (+) \\ \text { Social facilitation } & ++ & - & - & +++ & ++ & - \\ \text { Cooperation } & ++ & - & - & +++ & ++ & - \\ \text { Competition } & - & - & - & - & - & - \\ \text { Recognition } & - & - & - & + & (+) & -\end{array}$

We agree with Cunningham et al. [7] who state that expert-moderated online social support groups with member-generated content may have the potential to motivate individuals toward seeking treatment.

\section{Conclusions and Discussion}

This paper provided a qualitative evaluation of the persuasiveness of six Web-based alcohol interventions. The selected sites represent the current state-of-the-art of Web-based 
alcohol interventions. The results of this study suggest that there is room for improvement in both designing and implementing Web-based interventions for alcohol problems. The evaluation showed that the evaluated Web-based alcohol interventions may not be very persuasive. However, all evaluated sites successfully demonstrated trustworthiness, expertise, and surface credibility.

Primary task support principles were utilized relatively poorly in many of the sites. Interestingly, and perhaps rather worryingly, tailoring was applied in only one of the sites. This finding implies that the interventions may be targeted to too broad an audience. It is reasonable to assume that there should be different approaches for different user groups. In other words, if the user feels the (intervention) content is not designed for her/his needs, there is a high possibility that the user will discontinue using the program.

There were also notable differences between the evaluated intervention sites. For example, some sites placed more emphasis on online social support than others. Many of the sites did not offer any online social support. In our view, providing (expertmoderated) support groups as a part of a technology-based intervention is a very important aspect of such interventions. There are various techniques (e.g. instant messaging, chat rooms, discussion forums, social networking) readily available to facilitate communication between peers. In anonymous online support groups, the participants may overcome the feeling of being stigmatized, and time and location are no longer obstacles for participation. We suggest that Web-based interventions and support groups should not be considered as substitutes, rather as supplements to traditional forms of treatment and peer support.

As a conclusion, the evaluated Web-based alcohol interventions applied the persuasion principles described in the PSD model on a moderate level.

There are some limitations to this study. First, the language of the evaluated sites selected for this study was limited to English, Finnish, and Swedish. Second, no outside evaluators were used, thus the evaluations were at least partially based on the authors' subjective views. Third, the evaluation of persuasiveness of software and information systems may be problematic even with a proper method. The results should be regarded as indicatory for future studies. Finally, the evaluation of the quality of actual information presented in the sites is critical but beyond the scope of this paper.

As technology-enabled self-help interventions for manifold needs and diverse population continue to emerge, there is an apparent need for an understanding of how the persuasiveness of the systems affects users' intended behavior. Effective face-to-face counseling interventions may not be directly translated into the Web environment. Designing persuasive systems requires an interdisciplinary team of professionals with a thorough understanding of the problem domain and the underpinning theories.

Much emphasis has been put on measuring and proving the effectiveness of various digital interventions. However, future research should not limit its focus on validating interventions through randomized controlled trials only. We suggest that the persuasiveness of the system should be considered concurrently with the feasibility and effectiveness of technology-based interventions. 


\section{References}

1. Bewick, B.M., Trusler, K., Barkham, M., et al.: The Effectiveness of Web-Based Interventions Designed to Decrease Alcohol consumption-A Systematic Review. Preventive Medicine 47, 17-26 (2008)

2. Bewick, B.M., Trusler, K., Mulhern, B., et al.: The Feasibility and Effectiveness of a WebBased Personalised Feedback and Social Norms Alcohol Intervention in UK University Students: A Randomised Control Trial. Addictive Behaviors 33, 1192-1198 (2008)

3. Brendryen, H., Kraft, P.: Happy Ending: A Randomized Controlled Trial of a Digital Multi-Media Smoking Cessation Intervention. Addiction 103, 478-484 (2008)

4. Christensen, H., Griffiths, K.M., Jorm, A.F.: Delivering Interventions for Depression by using the Internet: Randomised Controlled Trial. British Medical Journal 328, 265 (2004)

5. Clarke, G., Eubanks, D., Reid, C.K., et al.: Overcoming Depression on the Internet $($ ODIN)(2): A Randomized Trial of a Self-Help Depression Skills Program with Reminders. Journal of Medical Internet Research 7, e16 (2005)

6. Copeland, J., Martin, G.: Web-Based Interventions for Substance use Disorders-A Qualitative Review. Journal of Substance Abuse Treatment 26, 109-116 (2004)

7. Cunningham, J.A., van Mierlo, T., Fournier, R.: An Online Support Group for Problem Drinkers: AlcoholHelpCenter. Net. Patient Education and Counseling 70, 193-198 (2008)

8. Duffett-Leger, L., Lumsden, J.: Interactive Online Health Promotion Interventions: A "health Check". In: IEEE International Symposium on Technology and Society (ISTAS 2008), Fredericton, New Brunswick, June 26-28, pp. 1-8 (2008)

9. Fogg, B.: Persuasive technology: Using computers to change what we think and do. Morgan Kaufmann, San Francisco (2002)

10. Hurling, R., Fairley, B.W., Dias, M.B.: Internet-Based Exercise Intervention Systems: Are More Interactive Designs Better? Psychology \& Health 21, 757-772 (2006)

11. IJsselsteijn, W.A., de Kort, Y.A.W., Midden, C., Eggen, B., van den Hoven, E.: Persuasive Technology for Human Well-being: Setting the Scene. In: IJsselsteijn, W., de Kort, Y., Midden, C., Eggen, B., van den Hoven, E. (eds.) PERSUASIVE 2006. LNCS, vol. 3962, pp. 1-5. Springer, Heidelberg (2006)

12. Koski-Jännes, A., Cunningham, J.: Interest in Different Forms of Self-Help in a General Population Sample of Drinkers. Addictive Behaviors 26, 91-99 (2001)

13. Koski-Jännes, A., Cunningham, J.A., Tolonen, K., et al.: Internet-Based Self-Assessment of drinking -3-Month Follow-Up Data. Addictive Behaviors 32, 533-542 (2007)

14. Linke, S., McCambridge, J., Khadjesari, Z., et al.: Development of a Psychologically Enhanced Interactive Online Intervention for Hazardous Drinking. Alcohol and Alcoholism 43, 669-674 (2008)

15. Linke, S., Murray, E., Butler, C., et al.: Internet-Based Interactive Health Intervention for the Promotion of Sensible Drinking: Patterns of use and Potential Impact on Members of the General Public. Journal of Medical Internet Research 9, e10 (2007)

16. Lustria, M.L.A., Cortese, J., Noar, S.M., et al.: Computer-Tailored Health Interventions Delivered Over the Web: Review and Analysis of Key Components. Patient Education and Counseling 74, 156-173 (2009)

17. Napolitano, M.A., Fotheringham, M., Tate, D., et al.: Evaluation of an Internet-Based Physical Intervention: A Preliminary Investigation. Annals of Behavioral Medicine 25, 92-99 (2003)

18. Oenema, A., Brug, J., Lechner, L.: Web-Based Tailored Nutrition Education: Results of a Randomized Controlled Trial. Health Education Research 16, 647-660 (2001) 
19. Oenema, A., Tan, F., Brug, J.: Short-Term Efficacy of a Web-Based Computer-Tailored Nutrition Intervention: Main Effects and Mediators. Annals of Behavioral Medicine 29, 54-63 (2005)

20. Oinas-Kukkonen, H., Harjumaa, M.: A Systematic Framework for Designing and Evaluating Persuasive Systems. In: Oinas-Kukkonen, H., Hasle, P., Harjumaa, M., Segerståhl, K. (eds.) PERSUASIVE 2008. LNCS, vol. 5033, pp. 164-176. Springer, Heidelberg (2008)

21. Oinas-Kukkonen, H., Harjumaa, M.: Towards Deeper Understanding of Persuasion in Software and Information Systems. In: The First International Conference on Advances in Human-Computer Interaction (ACHI 2008), Best Paper Award, Sainte Luce, Martinique, February 10-15, pp. 200-205 (2008)

22. Oinas-Kukkonen, H., Harjumaa, M.: Persuasive Systems Design: Key Issues, Process Model, and System Features. Communications of the Association for Information Systems 24, Article 28 (2009)

23. Portnoy, D.B., Scott-Sheldon, L.A.J., Johnson, B.T., et al.: Computer-Delivered Interventions for Health Promotion and Behavioral Risk Reduction: A Meta-Analysis of 75 Randomized Controlled Trials, 1988-2007. Preventive Medicine 47, 3-16 (2008)

24. Riper, H., Kramer, J., Smit, F., et al.: Web-Based Self-Help for Problem Drinkers: A Pragmatic Randomized Trial. Addiction 103, 218-227 (2008)

25. Ritterband, L.M., Gonder-Frederick, L.A., Cox, D.J., et al.: Internet Interventions: In Review, in use, and into the Future. Professional Psychology: Research and Practice 34, 527-534 (2003)

26. Room, R., Babor, T., Rehm, J.: Alcohol and Public Health. The Lancet 365, 519-530 (2005)

27. Strecher, V.J., Shiffman, S., West, R.: Randomized Controlled Trial of a Web-Based Computer-Tailored Smoking Cessation Program as a Supplement to Nicotine Patch Therapy. Addiction 100, 682-688 (2005)

28. Tørning, K., Oinas-Kukkonen, H.: Persuasive System Design: State of Art and Future Directions. In: Proceedings of the Fourth International Conference on Persuasive Technology, Claremont, CA, USA, April 26-29 (2009)

29. Wantland, D.J., Portillo, C.J., Holzemer, W.L., et al.: The Effectiveness of Web-Based Vs. Non-Web-Based Interventions: A Meta-Analysis of Behavioral Change Outcomes. Journal of Medical Internet Research 6, e40 (2004)

30. West, R.: Theory of addiction. Wiley-Blackwell, Oxford (2006)

31. Zetterqvist, K., Maanmies, J., Ström, L., et al.: Randomized Controlled Trial of InternetBased Stress Management. Cognitive Behaviour Therapy 32, 151-160 (2003) 